
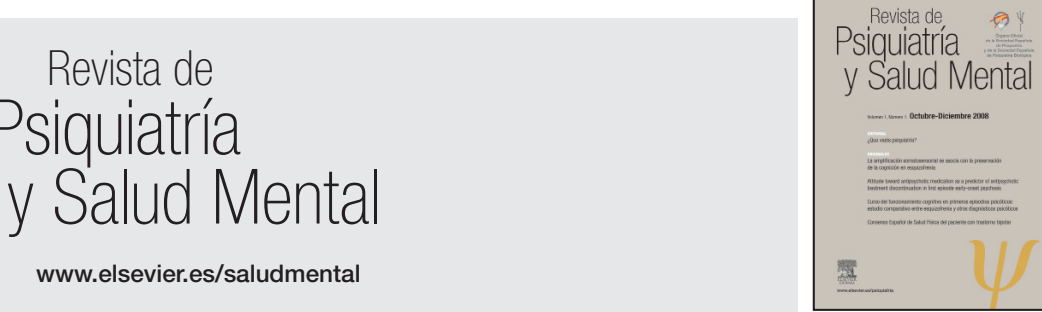

Originales

\title{
Consenso Español de Salud Física del Paciente con Trastorno Bipolar
}

\section{Julio Bobes ${ }^{\mathrm{a}}$, Jerónimo Sáiz Ruiz ${ }^{\mathrm{b}}$, José Manuel Montes ${ }^{\mathrm{c}, *}$, José Mostaza ${ }^{\mathrm{d}}$, Fernando Rico- Villademoros ${ }^{\mathrm{e}}$ y Eduard Vietaf; en representación del Grupo de Expertos para el Consenso de la Salud Física del Paciente con Trastorno Bipolar}

a Departamento de Medicina, Universidad de Oviedo, CIBERSAM, Oviedo; ${ }^{b}$ Departamento de Psiquiatría, Hospital Ramón y Cajal, Universidad de Alcalá, CIBERSAM, Madrid; 'Servicio de Psiquiatría, Hospital del Sureste, Madrid; 'Departamento de Medicina Interna, Hospital Carlos III, Madrid; eUniversidad de Alcalá de Henares, Madrid; 'Instituto Clínico de Neurociencias, Hospital Clínico de Barcelona, Universidad de Barcelona, CIBERSAM, Barcelona. España.

Recibido el 23 de septiembre de 2008; aceptado el 7 de octubre de 2008

\author{
PALABRAS CLAVE \\ Trastorno bipolar. \\ Salud física. \\ Consenso. \\ Morbilidad. \\ Mortalidad.
}

\begin{abstract}
Resumen
Introducción y objetivos: Los pacientes con trastorno bipolar presentan una morbilidad física y una mortalidad muy superior a la de la población general. Además de una mayor mortalidad por suicidio, tienen también una mayor prevalencia de enfermedades físicas.

El objetivo de este consenso, promovido por las Sociedades Españolas de Psiquiatría y Psiquiatría Biológica, en colaboración con las sociedades de médicos de asistencia primaria, es establecer recomendaciones prácticas sobre los procedimientos de detección, prevención e intervención en las enfermedades somáticas que coexisten con el trastorno bipolar para mejorar la calidad y esperanza de vida de estos pacientes.

Método: Las Sociedades Españolas de Psiquiatría y Psiquiatría Biológica eligieron un Comité Científico que seleccionó a su vez a 32 psiquiatras expertos y 10 médicos expertos en otras especialidades médicas. Se crearon grupos de trabajo para cada especialidad con la finalidad de adaptar las guías aplicadas en la población general a pacientes con trastorno bipolar. Partiendo de una revisión sistemática sobre la comorbilidad médica y la mortalidad en el trastorno bipolar se realizaron dos reuniones para acordar el consenso.

Resultados: En la revisión bibliográfica se detectó un riesgo aumentado, entre los pacientes con trastorno bipolar, de presentar hipertensión arterial, obesidad, tabaquismo, enfermedades pulmonares, migraña e infección por virus de la inmunodeficiencia humana (VIH). También se encontró evidencia de un aumento de mortalidad por enfermedades cardiovasculares, respiratorias e infecciones, además del suicidio. El grupo de expertos alcanzó el consenso en una serie de medidas básicas para la detección de comorbilidad médica aplicables a la monitorización de estos pacientes. Las recomendaciones resultantes serán asumidas y divulgadas por las sociedades promotoras.

Conclusiones: El decálogo generado en el Consenso Español de Salud Física del Paciente con Trastorno Bipolar recoge los aspectos más relevantes para la mejora del funcionamiento psicosocial, la calidad y la esperanza de vida en los pacientes con esta patología.
\end{abstract}


KEY WORDS

Bipolar disorder. Physical health. Consensus. Morbidity. Mortality.

\section{Spanish Consensus on Physical Health of Patients with Bipolar Disorder}

\begin{abstract}
Background and objective: Patients with bipolar disorder have much higher physical morbidity and mortality rates than the general population. In addition to a higher mortality rate from suicide, these patients also have a higher prevalence of physical disorders.

The aim of this consensus, promoted by the Spanish Societies of Psychiatry and Biological Psychiatry in collaboration with the General Practitioners' Societies, was to establish practical recommendations on procedures for detection, prevention and intervention in the somatic diseases that coexist with bipolar disorder in order to improve the quality of life and life expectancy of these patients.

Method: The Spanish Societies of Psychiatry and Biological Psychiatry designated a scientific committee that selected 32 expert psychiatrists and 10 experts from other medical specialties. Working groups were formed for each specialty for the purpose of adapting the guidelines applied in the general population to patients with bipolar disorder. A systematic review of medical comorbidity and mortality in these patients was carried out and two multidisciplinary consensus meetings were held.

Results: The literature review revealed an increased risk of hypertension, obesity, smoking, pulmonary diseases, migraine and HIV infection among patients with bipolar disorder. There was also evidence of higher mortality rates from cardiovascular and respiratory diseases and infections, as well as from suicide. The expert group reached a consensus on a series of basic measures to detect medical comorbidity applicable to the monitoring of these patients. The resulting recommendations will be accepted and disseminated by the promoting societies.

Conclusions: The recommendations generated by the Spanish Consensus on the Physical Health of Patients with Bipolar disorder include the most important aspects for the improvement of the psychosocial functioning, quality of life and life expectancy of these patients.
\end{abstract}

(c) 2008 Sociedad Española de Psiquiatría y Sociedad Española de Psiquiatría Biológica.

\section{Introducción}

El trastorno bipolar es una enfermedad mental grave que puede llegar a afectar a entre el 2 y el $5 \%$ de la población ${ }^{1,2}$. Supone un gran impacto sobre el funcionamiento del paciente que lo presenta, y ocupa el sexto lugar entre todas las enfermedades como causa global de discapacidad ${ }^{3}$.

Además, hay una evidencia creciente respecto al hecho de que los pacientes con trastorno bipolar presentan una morbilidad física y mortalidad superior a la de la población general. La mortalidad se encuentra duplicada, fundamentalmente por suicidio, y es 10 veces superior a la de la población general, pero también por otros factores como el incremento del riesgo de síndrome metabólico y enfermedades cardiovasculares que presentan estos pacientes ${ }^{4-7}$.

Aunque está poco estudiado, los datos disponibles sugieren que diversas enfermedades médicas (cardiovasculares, metabólicas, infecciosas, neurológicas y respiratorias) presentan una prevalencia más elevada en estos pacientes ${ }^{8,9}$. Las razones que contribuyen a esta mayor prevalencia son variadas. En primer lugar, las dificultades derivadas de un acceso adecuado a los servicios de prevención y tratamiento primarios de salud. Los hábitos de vida, con mayor consumo de sustancias adictivas, una vida sedentaria o la realización de dietas inadecuadas, representan también un factor de riesgo. Además, la presencia de síntomas afectivos se ha asociado a una activación inmunoinflamatoria que implica un mayor daño orgánico a través de la denominada carga alostática ${ }^{10}$. Por último, algunos de los psicofármacos empleados en su tratamiento pueden presentar efectos adversos asociados que repercuten sobre la salud física ${ }^{11}$.

Por otra parte, además de los riesgos que representan para la salud física del paciente, la presencia de estas enfermedades somáticas contribuye también a ensombrecer el pronóstico de la enfermedad bipolar, dificultan su tratamiento y recuperación, y empeoran la calidad de vida ${ }^{12}$. En definitiva, se puede decir que la presencia de los síntomas psiquiátricos no es la única causa de la repercusión global funcional que genera el trastorno bipolar sobre el paciente que lo padece.

Sin embargo, hay una tendencia a infradiagnosticar e infratratar las enfermedades médicas entre los pacientes psiquiátricos en general y entre los pacientes bipolares en particular, 13-15. De esta situación se deriva la consecuencia de sensibilizar a todos los profesionales de la salud sobre la necesidad de prevenir y tratar adecuadamente las enfermedades físicas en estos pacientes ${ }^{16}$. 
El objetivo del siguiente proyecto ha sido llevar a cabo un Consenso sobre la evaluación de la salud física y problemas médicos en el paciente con trastorno bipolar a lo largo de su vida, de forma similar al realizado recientemente por las Sociedades Españolas de Psiquiatría y Psiquiatría Biológica sobre la salud física del paciente con esquizofrenia ${ }^{17}$, estableciendo recomendaciones sobre los procedimientos diagnósticos y las intervenciones asistenciales necesarias para detectar y modificar factores de riesgo somáticos que tienen impacto sobre el funcionamiento psicosocial, la calidad y esperanza de vida de estos pacientes en España. Este consenso viene a unirse a otros consensos promovidos por otras sociedades científicas en el resto del mundo ${ }^{18,19}$.

\section{Métodos}

Este documento de consenso ha sido impulsado y avalado por las Sociedades Españolas de Psiquiatría (SEP) y de Psiquiatría Biológica (SEPB), en colaboración con la Sociedad Española de Medicina Rural y Generalista (SEMERGEN) y la de Medicina de Familia y Comunitaria (semFYC).

\section{Proceso de desarrollo}

El documento de consenso se desarrolló siguiendo las siguientes acciones:

- Las Sociedades Españolas de Psiquiatría y Psiquiatría Biológica eligieron un Comité Científico formado por 4 miembros y un Coordinador que seleccionaron a su vez a 32 psiquiatras expertos y 10 médicos expertos en otras especialidades médicas.

- Revisión sistemática de la literatura médica especializada sobre la comorbilidad médica y la mortalidad en el trastorno bipolar.

- Revisión de las guías nacionales e internacionales de prevención, diagnóstico y tratamiento de las distintas enfermedades identificadas como relevantes para el paciente con trastorno bipolar por parte de los médicos especialistas expertos asesores, tras la consiguiente adaptación a las peculiaridades de este trastorno.

- Dos reuniones de consenso multidisciplinarias.

- Redacción del documento de consenso final.

\section{Revisión sistemática de la literatura médica}

El objetivo de la revisión sistemática de la literatura médica fue valorar la incidencia, prevalencia o riesgo de enfermedades médicas, así como de la mortalidad, entre los pacientes con trastorno bipolar.

Se realizó una búsqueda bibliográfica en la base de datos Medline desde 1966 hasta enero de 2008. La búsqueda se limitó a estudios publicados en español o inglés. Además, se realizó una búsqueda en los listados de referencias de todos los artículos primarios identificados y de artículos de revisión relevantes.
Revisión de las guías nacionales e internacionales por parte de los médicos especialistas expertos asesores

En paralelo a la revisión sistemática de la literatura médica, los médicos especialistas expertos asesores revisaron las guías y documentos nacionales e internacionales sobre la prevención, diagnóstico y tratamiento de las enfermedades identificadas preliminarmente como relevantes para el trastorno bipolar en la revisión sistemática de la literatura médica.

\section{Reuniones de consenso multidisciplinarias}

Hubo dos reuniones de consenso. Ambas reuniones fueron multidisciplinarias y asistieron los miembros del Comité Científico, los representantes de las sociedades médicas y de la Universidad de Alcalá de Henares, 11 expertos asesores de las siguientes especialidades médicas: atención primaria (3 expertos), ginecología ( 2 expertos), enfermedades infecciosas (1 experto), endocrinología (1 experto), sistema circulatorio (1 experto), farmacología (1 experto), manejo de adicciones (1 experto) y prevención de la mortalidad (1 experto psiquiatra); y un grupo de 31 psiquiatras expertos participantes en la elaboración del consenso.

En la primera reunión, los miembros del Comité Científico realizaron una presentación sobre el estado actual del problema de salud física en los pacientes con trastorno bipolar. Posteriormente, cada uno de los médicos especialistas expertos asesores hizo una revisión de las guías internacionales y nacionales para el diagnóstico y seguimiento de cada una de las enfermedades prevalentes seleccionadas y luego se creó un grupo de trabajo para cada especialidad constituido por el médico especialista experto asesor correspondiente y varios de los psiquiatras expertos participantes. Cada grupo elaboraba un documento con las recomendaciones sobre las intervenciones diagnósticas, de monitorización, preventivas y terapéuticas adaptadas para los pacientes con trastorno bipolar.

En la segunda reunión se consensuaron por parte de todos los participantes las recomendaciones específicas generadas por cada grupo de trabajo que debían incorporarse al documento de consenso final.

\section{Resultados}

Revisión sistemática de la literatura médica Aunque los resultados de la revisión sistemática serán objeto de ulteriores publicaciones, a continuación se resumen las conclusiones más relevantes extraídas de la misma.

\section{Patología cardiovascular}

Número de estudios: 14 estudios controlados ${ }^{20-33}$ y 6 no controlados $^{34-39}$.

Hallazgos: aunque 2 estudios muestran resultados discrepantes, la mayoría de los estudios comparativos señalan que el trastorno bipolar se asocia a una frecuencia aumentada de 
hipertensión arterial (HTA), si bien con cifras de prevalencia puntual que varían mucho de unos estudios a otros (del 4,6 al $60,8 \%$ ). Además, se ha comunicado un mayor riesgo de HTA en pacientes con trastorno bipolar que en pacientes con esquizofrenia (odds ratio [OR] = 1,13, intervalo de confianza [IC] del 95\%, 1,01-1,26).

No hay pruebas que avalen de forma consistente que el trastorno bipolar se asocie a otras enfermedades cardiovasculares distintas de la hipertensión arterial. Lo mismo se puede decir respecto a enfermedades cerebrovasculares.

\section{Patología pulmonar}

Número de estudios: 9 estudios controlados $20,23,24,26-28,30,33,40 \mathrm{y}$ 3 no controlados $34,36,37$.

Hallazgos: se ha encontrado un riesgo de asma significativamente mayor en pacientes con trastorno bipolar en comparación con la población general. Las cifras de prevalencia varían entre el 3 y el $17 \%$ en los pacientes con trastorno bipolar frente al $2-10 \%$ en población general o el $8 \%$ en otras poblaciones de referencia. La prevalencia vida de asma en pacientes con trastorno bipolar (16,7\%; IC del 95\%, 15,1$18,4)$ también es significativamente superior ${ }^{23}$ a la observada en población general (9,7\%; IC del 95\%, 9,1-10,3).

También se encuentra en los pacientes bipolares un riesgo aumentado de sufrir enfermedad pulmonar obstructiva crónica (EPOC) con respecto a la población general (prevalencia vida del 2,7\% [IC del 95\%, 1,9-3,7] frente a 1,1\% [IC del $95 \%, 0,8-1,3])$.

\section{Patología gastrointestinal}

Número de estudios: 5 estudios controlados $20,26,28,30,41$ y 3 no controlados $^{36-38}$.

Hallazgos: aunque existen pocos datos, en 3 estudios controlados se sugiere que los pacientes con trastorno bipolar tienen un riesgo más elevado de presentar úlcera péptica. La prevalencia de úlcera péptica en estudios controlados y no controlados fue $0,9-10,8 \%$ en pacientes con trastorno bipolar, frente al 0,2-5,0\% en las poblaciones de referencia.

Aunque la información disponible sobre los trastornos hepáticos es también limitada, en 2 estudios controlados los pacientes con trastorno bipolar presentan un riesgo mayor de sufrir hepatopatías (OR = 3,97; IC del 95\%, 2,84-5,55).

\section{Patología endocrinometabólica}

Número de estudios: 17 estudios controlados $20,24,26-28,30-33,35,42-48$ y 13 no controlados ${ }^{34-39,49-55}$.

Hallazgos: en 6 de 7 de los estudios controlados que evalúan la prevalencia de obesidad se detectan unas cifras en pacientes con trastorno bipolar de entre el 19 y el 53\%, significativamente superior a la encontrada en la población general $(9-14 \%)$.

Se ha detectado también en algunos estudios un aumento de la prevalencia de diabetes, si bien con diferencias escasamente relevantes desde el punto de vista clínico. Los datos relativos a las dislipemias en pacientes con trastorno bipolar son muy variables y poco sólidos. Tan sólo un estudio contro- lado ha evaluado la presencia de síndrome metabólico en estos sujetos, y ha encontrado una prevalencia similar respecto a la de pacientes con esquizofrenia (22,5 frente a 29,7\%; p $=\mathrm{NS})^{31}$. Esta prevalencia de síndrome metabólico es muy similar $(22,4-24,7 \%)$ a la encontrada en estudios realizados en pacientes con trastorno bipolar en nuestro medio ${ }^{39,56}$.

En relación con el hipotiroidismo, cuya asociación con el trastorno bipolar ha sido muy debatida, tan sólo un estudio comparativo frente a población general abordó esta cuestión ${ }^{28}$. En este estudio, la prevalencia de hipotiroidismo en pacientes con trastorno bipolar fue del 9,6\% en comparación con el $2,5 \%$ en una muestra clínica sin trastorno bipolar $(O R=2,57 ; \mathrm{IC}$ del 95\%, 2,27-2,91; $\mathrm{p}<0,0001)$. Este riesgo parece aumentar entre los pacientes con ciclación rápida $(\mathrm{OR}=3,14$; IC del 95\%, 1,12-8,79; $\mathrm{p}<0,002)$ con respecto a los que no la presentan ${ }^{42}$. Si se incluyen otros estudios no comparativos, las cifras de prevalencia de hipotiroidismo en pacientes bipolares parecen situarse alrededor del $10 \%$.

\section{Patología renal y genitourinaria}

Número de estudios: 3 estudios controlados ${ }^{26,28,33}$ y 3 no controlados ${ }^{36-38}$.

Hallazgos: el riesgo de presentar nefropatías parece estar aumentado en los pacientes con trastorno bipolar respecto a pacientes no psiquiátricos $(\mathrm{OR}=2,78$; IC del 95\%, 1,87-4,14; $\mathrm{p}<0,0001)$.

\section{Patología musculoesquelética y del tejido conjuntivo}

Número de estudios: 6 estudios controlados $20,23,26,28,30,35$ y 4 no controlados ${ }^{35-38}$.

Hallazgos: no parece haber una enfermedad asociada al trastorno bipolar.

\section{Patología infecciosa}

Número de estudios: 5 estudios controlados ${ }^{26,28,57-59}$ y 3 no controlados $^{36,37,60}$.

Hallazgos: hay una relación sólida entre el trastorno bipolar y la infección por virus de la inmunodeficiencia humana (VIH). Las cifras de prevalencia oscilan en los estudios entre el 0,1 y $2,6 \%$, si se exceptúan los estudios realizados en países africanos con prevalencias de infección más elevadas.

\section{Patología neurológica}

Número de estudios: 11 estudios controlados $20,23,24,26,28,30,61-65$ y 10 no controlados $34,36-38,49,66-70$.

Hallazgos: la prevalencia de migraña en pacientes con trastorno bipolar es significativamente más elevada que en la población general (OR: 2,54; IC 95\%: 1,59-4,05), es mayor en mujeres y alcanza una media del $25 \%$ de prevalencia.

\section{Patología oncológica y hematológica}

Número de estudios: 3 estudios controlados $26,28,71$ y 2 no controlados $^{36,37}$.

Hallazgos: no hay datos que avalen la existencia de un incremento del cáncer en el trastorno bipolar. 


\section{Patología dermatológica, ocular,} otorrinolaringológica y dental

Número de estudios: 3 estudios no controlados de patología dermatológica ${ }^{36-38}$, un estudio controlado de patología ocu$\operatorname{lar}^{30}$ y ningún estudio sobre patologías otorrinolaringológica y dental.

Hallazgos: no hay datos para apoyar una asociación con estas enfermedades.

\section{Patología ginecológica y obstétrica}

Número de estudios: 3 estudios controlados ${ }^{20,28,72}$ y uno no controlado ${ }^{36}$.

Hallazgos: entre las mujeres con trastorno bipolar existe un riesgo aumentado de complicaciones gestacionales $(\mathrm{OR}=$ 1,23; IC del 95\%, 1,06-1,44), principalmente de placenta previa $(O R=2,13$; IC del 95\%, 1,15-3,94).

\section{Otros trastornos médicos}

Número de estudios: 6 estudios controlados ${ }^{23,24,26-30}$ y uno no controlado ${ }^{37}$.

Hallazgos: los pacientes con trastorno bipolar tienen una prevalencia de cuadros alérgicos significativamente superior a la de la población general, que alcanza cifras entre el 25 y el $40 \%$.

También se asocia con el síndrome de fatiga crónica (4 frente a $1 \%$ en la población general) y con el síndrome de múltiples sensibilidades químicas ( 4,6 frente $2,3 \%$ en la población general; $\mathrm{p}<0,05$ ).

\section{Trastornos relacionados con el uso de sustancias adictivas}

Número de estudios: 10 estudios controlados ${ }^{21,24-26,28,31,33,64,73,74}$ y uno no controlado ${ }^{70}$.

Dado que el objetivo de la revisión estaba focalizado en la comorbilidad médica, sólo se incluyen en este apartado los artículos que, con dicha orientación de búsqueda, incluían datos relativos al uso de sustancias adictivas.

Hallazgos: los pacientes con trastorno bipolar presentan un riesgo de abuso o dependencia de nicotina más de dos veces superior al de la población general $(\mathrm{OR}=2,1$; IC del $95 \%, 1,2-4,0)$, con una prevalencia de tabaquismo que se sitúa entre el 50 y el $60 \%$.

De la misma forma, el riesgo de abuso o dependencia de alcohol $(O R=19,63$; IC del $95 \%, 17,59-21,90)$ y de otras sustancias (OR $=42,91$; IC del 95\%, 37,83-48,66) también está muy elevado.

\section{Mortalidad \\ Mortalidad global (por cualquier causa) \\ Número de estudios: 6 estudios $4,6,75-78$. \\ Hallazgos: la mortalidad entre los pacientes con trastorno bipolar está incrementada al menos 2 veces en los hombres y 3 veces en las mujeres.}

Mortalidad por causas no naturales: Número de estudios: 10 estudios $4,6,75-82$.
Hallazgos: la mortalidad por suicidio está aumentada más de diez veces con respecto a la población general (razón estandarizada de mortalidad [REM] entre 10 y 18).

La mortalidad por accidentes también estaría aumentada hasta tres veces.

\section{Mortalidad por causas naturales}

Número de estudios: 5 estudios $75,76,79,80,83$.

Hallazgos: la mortalidad por causa cardiovascular está significativamente aumentada, exceso de mortalidad que puede alcanzar el $50 \%$. No parece haber aumento de mortalidad por enfermedad cerebrovascular o cáncer.

Por último, otras razones de aumento de la mortalidad que están incrementadas serían las derivadas de infecciones - que puede llegar a duplicar la esperable - y la mortalidad de causa respiratoria (REM entre 3 y 7 ).

\section{Algoritmos de monitorización e intervención sobre los problemas de salud física del paciente con trastorno bipolar}

Teniendo en cuenta los hallazgos obtenidos en la revisión sistemática respecto a la comorbilidad de trastornos físicos en los pacientes con trastorno bipolar, a continuación se recogen las recomendaciones consensuadas entre el Comité de Médicos Especialistas Expertos Asesores y los psiquiatras participantes sobre las intervenciones diagnósticas, preventivas y terapéuticas respecto a la salud física de los pacientes con trastorno bipolar. Todas estas intervenciones aparecen resumidas en la tabla 1 (protocolo de monitorización de la salud física en el paciente con trastorno bipolar). En todos los pacientes se debe realizar una anamnesis completa que incluya antecedentes familiares y personales médicos y psiquiátricos, incluyendo suicidalidad, consumo de sustancias adictivas, utilización de medicación concomitante e información sobre dieta y estilo de vida. A su vez se les debe preguntar sobre la presencia de disfunción sexual y deseo reproductivo. Se les deberá realizar una exploración general y una exploración analítica dirigidas a evaluar determinados trastornos específicos que se detallan a continuación.

\section{Patología cardiovascular}

Medidas de diagnóstico: la determinación de la presión arterial sistólica (PAS) y diastólica (PAD) debe realizarse de forma basal y en cada visita posterior. Si la presión arterial es normal y las visitas frecuentes se recomienda al menos una nueva determinación a los 3 meses y posteriormente cada 6 meses. Cuando se obtengan valores superiores o iguales a $140 / 90 \mathrm{mmHg}$, se debe repetir la medición hasta tres veces a intervalos de 2-3 min. Para realizar el diagnóstico de HTA se necesita repetir las mediciones en otros 2 días diferentes (triple toma) salvo que los valores en una única ocasión sean superiores a $189 / 110 \mathrm{mmHg}$.

Medidas de prevención: a todos los pacientes se debe recomendar dieta pobre en sal y realizar ejercicio adecuado a sus condiciones físicas. Es fundamental la reducción de peso si existe sobrepeso/obesidad. 
Medidas terapéuticas: si las cifras de presión arterial no están controladas $(\geq 140 / 90 \mathrm{mmHg}$ o menor de estas cifras en sujetos con diabetes o insuficiencia renal) se debe derivar a su médico de atención primaria. En este caso es importante indicar si está realizando tratamiento antipsicótico y cuál, para que se pueda decidir el hipotensor adecuado a cada caso. Si se realiza tratamiento con sales de litio hay que informar de las posibles interacciones con algunos fármacos hipotensores (en concreto diuréticos y antihipertensivos que actúan sobre el sistema renina-angiotensina).

\section{Patología endocrinometabólica}

Medidas de diagnóstico: de forma basal se debe determinar el peso, talla, índice de masa corporal (IMC = peso / talla en $\mathrm{m}^{2}$ ) y la medición del perímetro abdominal y realizar determinaciones analíticas de glucosa, colesterol total, colesterol unido a las lipoproteínas de alta densidad (cHDL), colesterol unido a las lipoproteínas de baja densidad ( $\mathrm{CLDL}$ ), triglicéridos, TSH y prolactina (sólo en caso de trastornos menstruales, disfunción sexual o población pediátrica). En todas las visitas se debe realizar determinación del peso (una al mes como máximo), así como la talla si el paciente está en período de crecimiento. El resto de determinaciones, si están dentro de la normalidad (IMC $<25 \mathrm{~kg} / \mathrm{m}^{2}, \mathrm{c}-\mathrm{HDL}$ en varones $\geq 40 \mathrm{mg} / \mathrm{dl}$ y en mujeres $\geq 50$ $\mathrm{mg} / \mathrm{dl}$, triglicéridos $\leq 150 \mathrm{mg} / \mathrm{dl}$, glucemia $<100 \mathrm{mg} / \mathrm{dl}$, TSH entre $0.3-6 \mathrm{mU} / \mathrm{l}$ y prolactina $<20 \mathrm{ng} / \mathrm{ml}$, se repetirán anualmente.

Si en una determinación aparecen cifras de glucemia entre 100 y $126 \mathrm{mg} / \mathrm{dl}$, de TSH superiores a $6 \mathrm{mU} / \mathrm{l}$ o de prolactina $>20 \mathrm{ng} / \mathrm{ml}$ se debe repetir la analítica. También repetir la TSH tras 3 o 6 meses de iniciar tratamiento con litio.

En el caso de presencia de síndrome metabólico (definido por la presencia de al menos 3 de los siguientes criterios: perímetro abdominal en varones $>102 \mathrm{~cm}$ y en mujeres $>88$ $\mathrm{cm}$; cHDL en varones < $40 \mathrm{mg} / \mathrm{dl}$ y en mujeres < $50 \mathrm{mg} / \mathrm{dl}$; PA $>$ 130/85 mmHg; triglicéridos > $150 \mathrm{mg} / \mathrm{dl}$; glucemia > 100 $\mathrm{mg} / \mathrm{dl}$ ) se deben monitorizar los parámetros cada 6 meses, así como si el paciente ha ganado peso (> 7\% del basal).

Medidas de prevención: a todos los pacientes se debe recomendar dieta y ejercicio, así como recordarles deben mantener hábitos de vida saludable.

Medidas terapéuticas: En el caso de dislipemia (colesterol total > $250 \mathrm{mg} / \mathrm{dl}$, triglicéridos $>200 \mathrm{mg} / \mathrm{dl}$ ), síndrome metabólico o diabetes (glucemia > $126 \mathrm{mg} / \mathrm{dl}$ ) se debe derivar al paciente a atención primaria, así como también valorar una alternativa al tratamiento farmacológico, en particular si se trata de fármacos que se asocien a aumento ponderal. En el caso de aumento de peso (> 7\% del basal) también se debe considerar el cambio de tratamiento farmacológico y la derivación al médico de atención primaria si este incremento de peso se acompaña de modificaciones negativas en los factores de riesgo cardiovascular de acuerdo con los criterios previamente comentados.

Cuando se mantienen cifras altas de TSH se debe valorar la derivación al endocrinólogo. También se debe valorar la derivación cuando la hiperprolactinemia no sea de causa farmacológica, tenga repercusión clínica o aparezcan cifras por encima de $200 \mathrm{ng} / \mathrm{ml}$.

\section{Patología infecciosa}

Medidas de diagnóstico: se deben realizar de forma basal y siempre que existan sospechas de prácticas de riesgo las siguientes determinaciones serológicas: anticuerpos anti- $\mathrm{VIH}$ (ELISA), anticuerpos anti-VHB (ELISA), anticuerpos anti-VHC (ELISA) y VDRL/RPR.

Medidas de prevención: se recomienda la vacuna para hepatitis $B$ en aquellos pacientes que no tienen evidencia de infección.

Medidas terapéuticas: en caso de positividad derivar al especialista correspondiente.

\section{Mujeres en edad fértil}

Medidas de prevención: se debe preguntar sobre el deseo reproductivo, realizando psicoeducación y favoreciendo un embarazo programado. Mientras no se desee el embarazo, asesorar sobre un método anticonceptivo adecuado, recomendando la utilización del preservativo si existen conductas sexuales de riesgo. En caso de uso de inductores del citocromo P450 (p. ej., carbamacepina, topiramato, oxcarbamacepina) usar dosis de estrógenos superiores o métodos contraceptivos alternativos así como ajustar dosis de lamotrigina (evaluando los valores plasmáticos) si se utilizan estrógenos. Hay que tratar de utilizar los psicofármacos con menor riesgo de teratogenia.

Medidas terapéuticas: en el caso de embarazo, debe realizarse una evaluación individualizada de beneficio-riesgo respecto a la conveniencia de continuar o no el tratamiento psicofarmacológico durante el primer trimestre del embarazo, informando de los posibles riesgos maternos y fetales. Se debe considerar como de alto riesgo, lo cual conlleva determinar los valores plasmáticos de todos los fármacos posibles (especialmente litio, valproato y carbamacepina), evaluar perfil de toxicidad hepatorrenal, descartar diabetes gestacional y alteración tiroidea, así como, en el caso de utilizar anticonvulsivantes o litio, evaluar morfología fetal, detección de malformaciones de tubo neural y cardiopatías (ecocardiografía fetal).

El tratamiento durante el embarazo se debe simplificar al máximo, considerando ajuste de dosis por hemodilución materna, durante el segundo y tercer trimestre del embarazo. Si la paciente recibe tratamiento con valproato y/o carbamacepina se debe suplementar con ácido fólico durante toda la gestación.

\section{Manejo de adicciones}

Medidas de diagnóstico: recoger de forma basal y en cada visita el uso de sustancias. Realizar hemograma para determinar volumen corpuscular medio (VCM) y perfil hepático como marcadores de consumo de alcohol.

Medidas de prevención: realizar psicoeducación respecto al riesgo asociado al consumo de sustancias tanto para la 


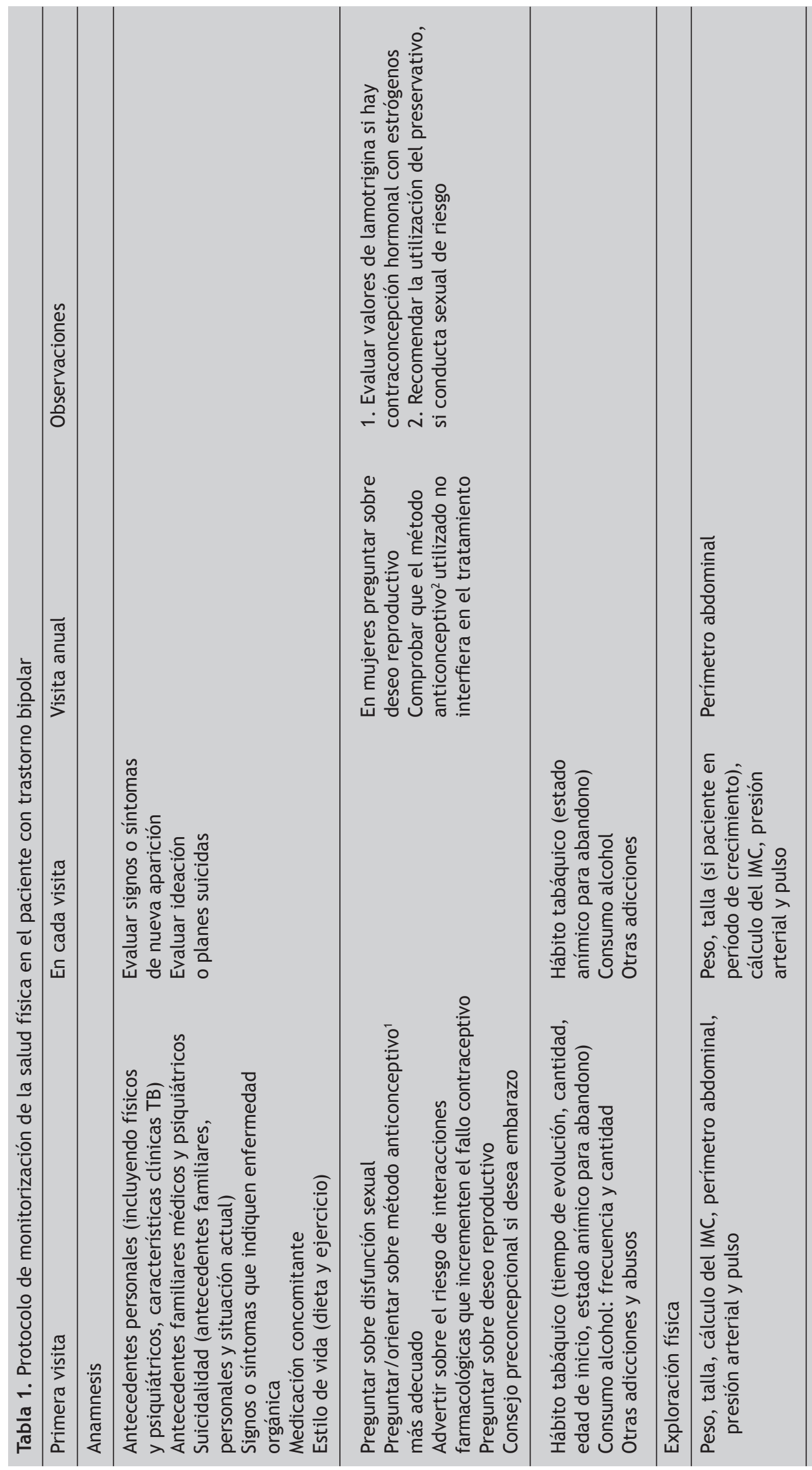




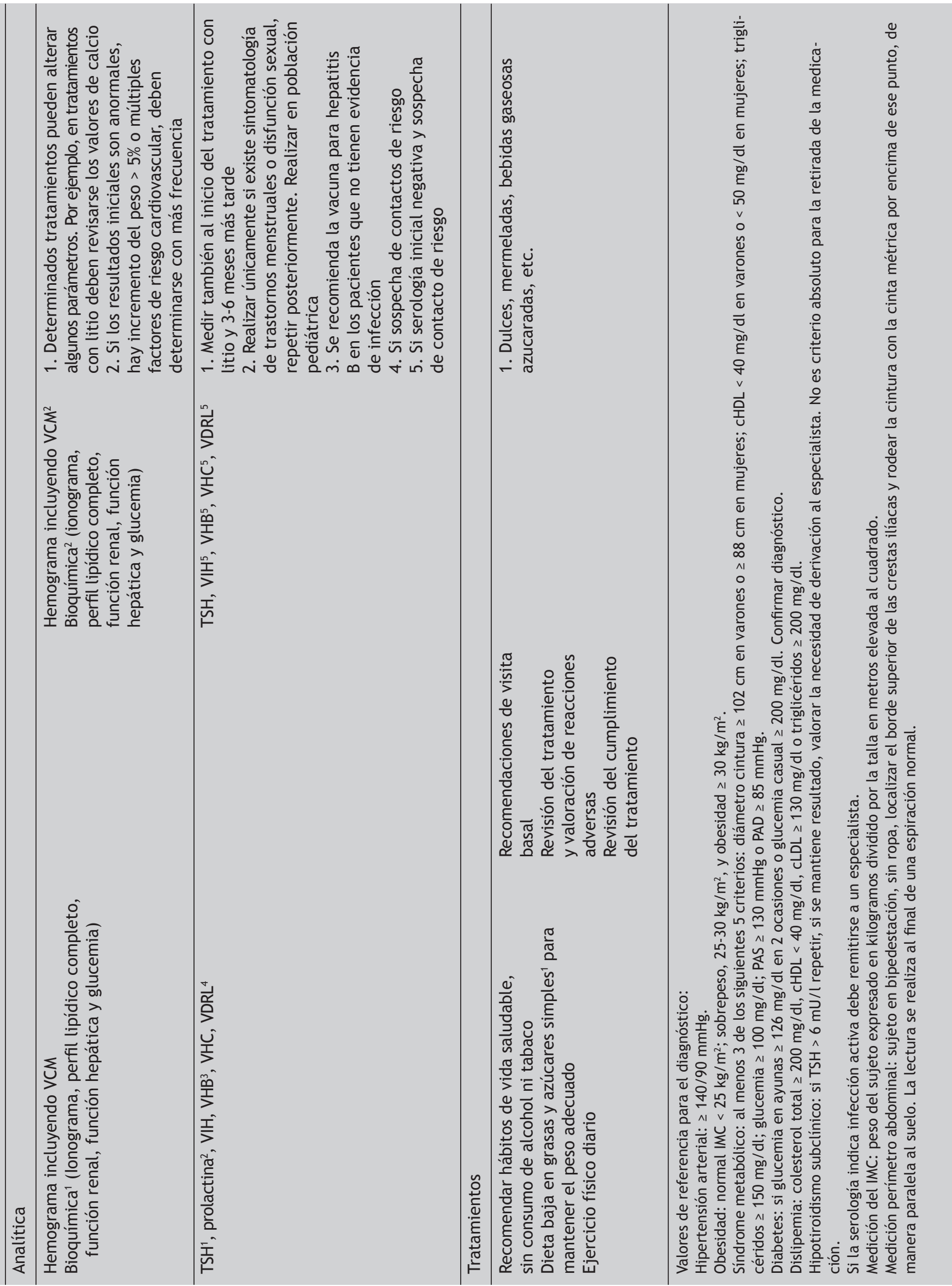


evolución de la enfermedad como en la posible interacción con el tratamiento.

Medidas terapéuticas: en caso de tabaquismo recomendar seguir tratamiento de deshabituación y los tratamientos sustitutivos de la nicotina como parches o chicles. Valorar la utilización de tratamientos con eficacia sobre el uso de sustancias, como el valproato en el caso del alcohol. Considerar derivación a centros de atención especializada en patología dual.

\section{Prevención mortalidad/suicidios}

Medidas de diagnóstico: se debe realizar un diagnóstico del trastorno bipolar de la forma más temprana posible, así como iniciar el adecuado tratamiento. Recoger la historia del trastorno teniendo en cuenta que determinados factores se asocian con mayor riesgo de suicidio (inicio temprano, polaridad depresiva o episodios mixtos, ciclación rápida, tipo II, etc.). Preguntar sobre antecedentes personales de intento de suicidio, exploración de antecedentes familiares de intentos de suicidio, así como de abuso de sustancias.

Medidas de prevención: explorar la ideación suicida en cada visita. Realizar ingreso cuando se precise.

\section{Otros trastornos médicos}

Medidas de diagnóstico: recoger historia personal de posible ulcus péptico, migraña, enfermedad respiratoria y alergias.

Medidas terapéuticas: tener en cuenta la posible interacción con el litio de los antiinflamatorios no esteroideos utilizados para el tratamiento sintomático de la migraña. También hay que vigilar la posible presencia o aparición de insuficiencia renal para ajustar los valores de litio. Los corticoides utilizados en el control del asma y otras enfermedades pulmonares pueden precipitar episodios afectivos.

\section{Decálogo de Consenso}

1. La Sociedad Española de Psiquiatría (SEP) y la Sociedad Española de Psiquiatría Biológica (SEPB) en colaboración con la Sociedad Española de Medicina Rural y Generalista (SEMERGEN) y la de Medicina de Familia y Comunitaria (se$\mathrm{mFYC}$ ) consideran que el trastorno bipolar se asocia a un notable deterioro de la salud física y discapacidad debido a factores inherentes a la propia enfermedad, a una menor concienciación de hábitos saludables y al efecto de algunos tratamientos.

2. La Estrategia Nacional y Europea de Salud Mental, así como la OMS, promueven la mejora del estado de salud física de los pacientes con enfermedades mentales y el empleo de medidas preventivas y de promoción de la salud.

3. Los pacientes con trastorno bipolar muestran mayor incidencia que la población general de enfermedad cardiovascular, metabólica, respiratoria, neurológica e infecciosa, y un mayor riesgo de mortalidad global (cardiovascular, respiratoria e infecciosa), además de presentar un mayor riesgo de suicidio.

4. Es necesario mejorar el acceso de los pacientes con trastorno bipolar a los recursos sanitarios, así como el grado de coordinación entre la asistencia especializada y primaria para el mejor cuidado de la salud psíquica y física de estos pacientes.

5. Se considera imprescindible contar con un historial clínico completo y terapéuticas integrales que incorporen la atención a los aspectos de salud física del paciente con trastorno bipolar.

6. Se recomiendan actuaciones (protocolo de monitorización de la salud física en el paciente con trastorno bipolar) dirigidas a la prevención, diagnóstico y manejo de las diferentes enfermedades concomitantes, factores y comportamientos de riesgo que se asocian al trastorno bipolar.

7. Se requiere un control y atención específica en los pacientes con trastorno bipolar más vulnerables como son los ancianos, los niños y adolescentes y las mujeres en edad fértil.

8. Los programas psicoeducativos incorporarán aspectos de promoción de la salud física y estilo de vida, además de los directamente relacionados con la salud mental y la prevención de recaídas.

9. Para mejorar el estado de salud física de los pacientes con trastorno bipolar, es necesaria la realización de formación continuada de los psiquiatras clínicos, los médicos de atención primaria, y otros profesionales sanitarios.

10. Finalmente, las sociedades promotoras (SEP, SEPB, SEMERGEN y semFYC) consideran necesaria la máxima difusión de este Consenso español para promover el cuidado de la salud física del paciente con trastorno bipolar como parte integral de su tratamiento.

\section{Conclusiones}

Este proyecto, promovido por las Sociedades Españolas de Psiquiatría (SEP) y de Psiquiatría Biológica (SEPB), en colaboración con la Sociedades Españolas de Medicina Rural y Generalista (SEMERGEN) y de Medicina de Familia y Comunitaria (semFYC), tiene como objetivo llevar a cabo un Consenso sobre la Evaluación de la Salud Física y problemas médicos en el paciente con trastorno bipolar durante su vida. El decálogo de consenso viene a recoger los puntos más relevantes a este respecto, con el propósito de que su difusión suponga un impacto sobre el funcionamiento psicosocial, la calidad y esperanza de vida de estos pacientes en España.

\section{Agradecimientos}

Queremos agradecer su colaboración a:

Comité científico: Julio Bobes (Oviedo); José Mostaza (Madrid); Jerónimo Saiz (Madrid); Eduard Vieta (Barcelona).

Miembros de la Sociedades Médicas: José Angel Arbesú (Oviedo, SEMERGEN); Ana Pastor (Madrid, semFYC).

Expertos no psiquiatras participantes: Cecilio Álamo (Madrid); M. Jesús Cerededo (A Coruña); Oriol Coll (Barcelona); 
Alejandro Cuadrado (Zamora); Sandra Hernández (Barcelona); Carlos Lahoz (Madrid); Silvia Mondón (Barcelona); Santiago Moreno (Madrid); Ana Pastor (Madrid); Fernando Rico-Villademoros (Universidad Alcalá de Henares, Madrid); Carmen Suárez (Madrid).

Psiquiatras: Enrique Baca (Madrid); Antonio Carrillo (Madrid); Alberto Cebollada (Madrid); Jorge Cervilla (Granada); José Manuel Crespo (Barcelona); Rosario De Arce (Madrid); Chelo De Dios (Madrid); Elena Ezquiaga (Madrid); Raúl Fernández-Villamor (Sevilla); Juan Franch (Valladolid); Aurelio García (Madrid); Paz García-Portilla (Oviedo); José Manuel Goikolea (Barcelona); Ana González-Pinto (Vitoria); M. ${ }^{a}$ Luisa Imaz (Barcelona); Lorenzo Livianos (Valencia); Gema Medina (Valladolid); Carmen Moreno (Madrid); Evaristo Nieto (Barcelona); José Manuel Olivares (Pontevedra); Josefina Pérez (Barcelona); Jesús Ramos (Madrid); Alfonso Rodríguez (Barcelona); José Luis Rodríguez (Madrid); Pilar Sáiz (Oviedo); Bernardo Sánchez (Barcelona); Susana Santamarina (Oviedo); Fernando Sarramea (Córdoba); Rafael Segarra (Bilbao); César Soutullo (Pamplona); Francisco Toledo (Murcia); Pedro Torres (Jaén); Jesús Valle (Madrid).

Este proyecto de consenso ha sido promovido por las Sociedades Españolas de Psiquiatría (SEP) y de Psiquiatría Biológica (SEPB), en colaboración con la Sociedad Española de Medicina Rural y Generalista (SEMERGEN) y la de Medicina de Familia y Comunitaria (semFYC) y ha sido copatrocinado y financiado conjuntamente por Bristol-Myers Squibb y Otsuka Pharmaceuticals.

\section{Bibliografía}

1. Angst J. The emerging epidemiology of hypomania and bipolar II disorder. J Affect Disord. 1998;50:143-51.

2. Merikangas KR, Akiskal HS, Angst J, Greenberg PE, Hirschfeld RM, Petukhova M, et al. Lifetime and 12-month prevalence of bipolar spectrum disorder in the National Comorbidity Survey replication. Arch Gen Psychiatry. 2007;64:543-52.

3. Murray CJL, Lopez AD, eds. The global burden of disease and injury series, volume 1: a comprehensive assessment of mortality and disability from diseases, injuries, and risk factors in 1990 and projected to 2020. Cambridge: Harvard University Press; 1996.

4. Angst F, Stassen HH, Clayton PJ, Angst J. Mortality of patients with mood disorders: follow-up over 34-38 years. J Affect Disord. 2002;68:167-81.

5. Baldessarini RJ, Tondo L. Suicide risk and treatments for patients with bipolar disorder. JAMA. 2003;290:1517-9.

6. Osby U, Brandt L, Correia N, Ekbom A, Sparén P. Excess mortality in bipolar and unipolar disorder in Sweden. Arch Gen Psychiatry. 2001;58:844-50.

7. Torrent C, Amann B, Sánchez-Moreno J, Colom F, Reinares M, Comes $\mathrm{M}$, et al. Weight gain in bipolar disorder: pharmacological treatment as a contributing factor. Acta Psychiatr Scand. 2008;118:4-18.

8. Garcia-Portilla MP, Saiz PA, Bascaran MT, Martínez S, Benabarre $A$, Sierra $P$, et al on behalf of the General Health Status in Bipolar Disorder Collaborative Group. Cardiovascular risk in patients with bipolar disorder. J Affect Dis. 2008. En prensa.

9. Mclntyre RS, Soczynska JK, Beyer JL, Woldeyohannes HO, Law CW, Miranda A, et al. Medical comorbidity in bipolar disorder: re-prioritizing unmet needs. Curr Opin Psychiatry. 2007;20:40616.

10. MCEwen BS. Mood disorders and allostatic load. Biol Psychiatry. 2003; 54:200-7.

11. Lieberman JA, Stroup TS, McEvoy JP, Swartz MS, Rosenheck RA, Perkins DO, et al; Clinical Antipsychotic Trials of Intervention Effectiveness (CATIE) Investigators. Effectiveness of antipsychotic drugs in patients with chronic schizophrenia. N Engl J Med. 2005;353:1209-23.

12. Black DW, Winokur G, Bell S, Nasrallah A, Hulbert J. Complicated mania. Comorbidity and immediate outcome in the treatment of mania. Arch Gen Psychiatry. 1988;45:232-6.

13. D’Ercole A, Skodol AE, Struening E, Curtis J, Millman J. Diagnosis of physical illness in psychiatric patients using axis III and a standardized medical history. Hosp Community Psychiatry. 1991;42:395-400.

14. Koranyi EK. Morbidity and rate of undiagnosed physical illnesses in a psychiatric clinic population. Arch Gen Psychiatry. 1979;36:414-9.

15. Kupfer DJ. The increasing medical burden in bipolar disorder. JAMA. 2005;293:2528-30.

16. Kilbourne AM, Post EP, Nossek A, Drill L, Cooley S, Bauer MS. Improving medical and psychiatric outcomes among individuals with bipolar disorder: a randomized controlled trial. Psychiatr Serv. 2008;59:760-8.

17. Saiz J, Bobes J, Vallejo J, Giner J, García-Portilla MP. Consenso sobre la salud física del paciente con esquizofrenia de las Sociedades Españolas de Psiquiatría y de Psiquiatría Biológica. Actas Esp Psiquiatr. 2008. En prensa.

18. Fleischhacker WW, Cetkovich-Bakmas M, De Hert M, Hennekens $\mathrm{CH}$, Lambert $\mathrm{M}$, Leucht $\mathrm{S}$, et al. Comorbid somatic illnesses in patients with severe mental disorders: clinical, policy, and research challenges. J Clin Psychiatry. 2008;69:514-9.

19. Yatham LN, Kennedy SH, O'Donovan C, Parikh S, MacQueen G, McIntyre R, et al. Canadian Network for Mood and Anxiety Treatments (CANMAT) guidelines for the management of patients with bipolar disorder: consensus and controversies. Bipolar Disord. 2005;7 Suppl 3:5-69.

20. Ghadirian AM, Engelsmann F. Somatic illness in manic-depressive and schizophrenic patients. J Psychosom Res. 1985;29:281-6.

21. Yates WR, Wallace R. Cardiovascular risk factors in affective disorder. J Affect Disord. 1987;12:129-34.

22. Winokur G, Coryell W, Endicott J, Akiskal H. Further distinctions between manic-depressive illness (bipolar disorder) and primary depressive disorder (unipolar depression) Am J Psychiatry. 1993;150:1176-81.

23. Calabrese JR, Hirschfeld RM, Reed M, Davies MA, Frye MA, Keck PE, et al. Impact of bipolar disorder on a U.S. community sample. J Clin Psychiatry. 2003;64:425-32.

24. Hirschfeld RM, Calabrese JR, Weissman MM, Reed M, Davies MA, Frye MA, et al. Screening for bipolar disorder in the community. J Clin Psychiatry. 2003;64:53-9.

25. Nilsson FM, Kessing LV. Increased risk of developing stroke for patients with major affective disorder--a registry study. Eur Arch Psychiatry Clin Neurosci. 2004;254:387-91.

26. Kilbourne AM, Cornelius JR, Han X, Pincus HA, Shad M, Salloum I et al. Burden of general medical conditions among individuals with bipolar disorder. Bipolar Disord. 2004;6:368-73.

27. Oedegaard KJ, Fasmer OB. Is migraine in unipolar depressed patients a bipolar spectrum trait? J Affect Disord. 2005;84:23342.

28. Carney CP, Jones LE. Medical comorbidity in women and men with bipolar disorders: a population-based controlled study. Psychosom Med. 2006;68:684-91.

29. Johannessen L, Strudsholm U, Foldager L, Munk-Jørgensen P. Increased risk of hypertension in patients with bipolar disorder and patients with anxiety compared to background 
population and patients with schizophrenia. J Affect Disord. 2006;95:13-7.

30. McIntyre RS, Konarski JZ, Soczynska JK, Wilkins K, Panjwani G, Bouffard B, et al. Medical comorbidity in bipolar disorder: implications for functional outcomes and health service utilization. Psychiatr Serv. 2006;57:1140-4.

31. Birkenaes AB, Opjordsmoen S, Brunborg C, Engh JA, Jonsdottir $\mathrm{H}$, Ringen PA, et al. The level of cardiovascular risk factors in bipolar disorder equals that of schizophrenia: a comparative study. J Clin Psychiatry. 2007;68:917-23.

32. Kilbourne AM, Brar JS, Drayer RA, Xu X, Post EP. Cardiovascular disease and metabolic risk factors in male patients with schizophrenia, schizoaffective disorder, and bipolar disorder. Psychosomatics. 2007;48:412-7.

33. Lin HC, Tsai SY, Lee HC. Increased risk of developing stroke among patients with bipolar disorder after an acute mood episode: a six-year follow-up study. J Affect Disord. 2007;100:4954.

34. Strakowski SM, Tohen M, Stoll AL, Faedda GL, Goodwin DC. Comorbidity in mania at first hospitalization. Am J Psychiatry. 1992;149:554-6.

35. McElroy SL, Frye MA, Suppes T, Dhavale D, Keck PE Jr, Leverich GS, et al. Correlates of overweight and obesity in 644 patients with bipolar disorder. J Clin Psychiatry. 2002;63:207-13.

36. Beyer J, Kuchibhatla M, Gersing K, Krishnan KR. Medical comorbidity in a bipolar outpatient clinical population. Neuropsychopharmacology. 2005;30:401-4.

37. Fenn HH, Bauer MS, Altshuler L, Evans DR, Williford WO, Kilbourne AM et al. VA Cooperative Study \#430 Team. Medical comorbidity and health-related quality of life in bipolar disorder across the adult age span. J Affect Disord. 2005;86:47-60.

38. Thompson WK, Kupfer DJ, Fagiolini A, Scott JA, Frank E. Prevalence and clinical correlates of medical comorbidities in patients with bipolar I disorder: analysis of acute-phase data from a randomized controlled trial. J Clin Psychiatry. 2006;67:783-8.

39. Garcia-Portilla MP, Saiz PA, Benabarre A, Sierra P, Perez J, Rodriguez $A$, et al. The prevalence of metabolic syndrome in patients with bipolar disorder. J Affect Disord. 2008;106:197201.

40. Strudsholm U, Johannessen L, Foldager L, Munk-Jørgensen P. Increased risk for pulmonary embolism in patients with bipolar disorder. Bipolar Disord. 2005;7:77-81.

41. Ewald H, Mortensen PB, Mors O. Decreased risk of acute appendicitis in patients with schizophrenia or manic-depressive psychosis. Schizophr Res. 2001;49:287-93.

42. Bauer MS, Whybrow PC, Winokur A. Rapid cycling bipolar affective disorder. I. Association with grade I hypothyroidism. Arch Gen Psychiatry. 1990;47:427-32.

43. Benazzi F. Clinical differences between bipolar II depression and unipolar major depressive disorder: lack of an effect of age. J Affect Disord. 2003;75:191-5.

44. Cassidy F, Ahearn E, Carroll BJ. Elevated frequency of diabetes mellitus in hospitalized manic-depressive patients. Am J Psychiatry. 1999; 156:1417-20.

45. Elmslie JL, Silverstone JT, Mann JI, Williams SM, Romans SE. Prevalence of overweight and obesity in bipolar patients. J Clin Psychiatry. 2000;61:179-84.

46. Lilliker SL. Prevalence of diabetes in a manic-depressive population Compr Psychiatry. 1980;21:270-5.

47. Regenold WT, Thapar RK, Marano C, Gavirneni S, Kondapavuluru PV. Increased prevalence of type 2 diabetes mellitus among psychiatric inpatients with bipolar I affective and schizoaffective disorders independent of psychotropic drug use. J Affect Disord. 2002;70:19-26.

48. Thomsen AF, Kessing LV. Increased risk of hyperthyroidism among patients hospitalized with bipolar disorder. Bipolar Disord. 2005;7:351-7.
49. Blehar MC, DePaulo JR Jr, Gershon ES, Reich T, Simpson SG, Nurnberger JI Jr. Women with bipolar disorder: findings from the NIMH Genetics Initiative sample. Psychopharmacol Bull. 1998;34:239-43.

50. Fagiolini A, Frank E, Houck PR, Mallinger AG, Swartz HA, Buysse DJ, et al. Prevalence of obesity and weight change during treatment in patients with bipolar I disorder. J Clin Psychiatry. 2002;63:528-33.

51. Fagiolini A, Frank E, Scott JA, Turkin S, Kupfer DJ. Metabolic syndrome in bipolar disorder: findings from the Bipolar Disorder Center for Pennsylvanians. Bipolar Disord. 2005;7:424-30.

52. Kupka RW, Nolem WA, Post RM, McElroy SL, Altshuler LL, Denicoff $\mathrm{KD}$, et al. High rate of autoimmune thyroiditis in bipolar disorder: lack of association with lithium exposure. Biol Psychiatry. 2002;51:305-11.

53. Valle J, Ayuso-Gutierrez JL, Abril A, Ayuso-Mateos JL. Evaluation of thyroid function in lithium-naive bipolar patients. Eur Psychiatry. 1999;14:341-5.

54. Ruzickova M, Slaney C, Garnham J, Alda M. Clinical features of bipolar disorder with and without comorbid diabetes mellitus. Can J Psychiatry. 2003;48:458-61.

55. Wang PW, Sachs GS, Zarate CA, Marangell LB, Calabrese JR, Goldberg JF, et al. Overweight and obesity in bipolar disorders. J Psychiatr Res. 2006;40:762-4.

56. Sicras A, Rejas J, Navarro R, Serrat J, Blanca M. Metabolic syndrome in bipolar disorder: a cross-sectional assessment of a Health Management Organization database. Bipolar Disord. 2008;10:607-16.

57. Beyer JL, Taylor L, Gersing KR, Krishnan KR. Prevalence of HIV infection in a general psychiatric outpatient population. Psychosomatics. 2007;48:31-7.

58. Dickerson FB, Boronow JJ, Stallings C, Origoni AE, Cole S, Krivogorsky $B$, et al. Infection with herpes simplex virus type 1 is associated with cognitive deficits in bipolar disorder. Biol Psychiatry. 2004;55:588-93.

59. Himelhoch S, McCarthy JF, Ganoczy D, Medoff D, Dixon LB, Blow FC. Understanding associations between serious mental illness and HIV among patients in the VA Health System. Psychiatr Serv. 2007;58:1165-72.

60. Nakimuli-Mpungu E, Musisi S, Mpungu SK, Katabira E. Primary mania versus HIV-related secondary mania in Uganda. Am J Psychiatry. 2006;163:1349-54.

61. Marchesi C, De Ferri A, Petrolini N, Govi A, Manzini GC, Coiro V, et al. Prevalence of migraine and muscle tension headache in depressive disorders. J Affect Disord. 1989;16:33-6.

62. Shulman KI, Tohen M, Satlin A, Mallya G, Kalunian D. Mania compared with unipolar depression in old age. Am J Psychiatry. 1992;149:341-5.

63. Fasmer $\mathrm{OB}$. The prevalence of migraine in patients with bipolar and unipolar affective disorders. Cephalalgia. 2001;21:894-9.

64. Kessing LV, Nilsson FM. Increased risk of developing dementia in patients with major affective disorders compared to patients with other medical illnesses. J Affect Disord. 2003;73:261-9.

65. Nilsson FM, Kessing LV, Bolwig TG. On the increased risk of developing late-onset epilepsy for patients with major affective disorder. J Affect Disord. 2003;76:39-48.

66. Low NC, Du Fort GG, Cervantes P. Prevalence, clinical correlatos, and treatment of migraine in bipolar disorder. Haedache. 2003;43:940-9.

67. Mahmood T, Romans S, Silverstone T. Prevalence of migraine in bipolar disorder. J Afffect Disrd. 1999;52:239-41.

68. Mukherjee S, Rosen AM, Caracci G, Shukla S. Persistent tardive dyskinesia in bipolar patients. Arch Gen Psychiatry. 1986;43:342-6.

69. Snowdon J. A retrospective case-note study of bipolar disorder in old age. Br J Psychiatry. 1991;158:485-90.

70. Sajatovic M, Blow FC, Ignacio RV. Psychiatric comorbidity in older adults with bipolar disorder. Int J Geriatr Psychiatry. 2006;21:582-7. 
71. Hippisley-Cox J, Vinogradova Y, Coupland C, Parker C. Risk of malignancy in patients with schizophrenia or bipolar disorder: nested case-control study. Arch Gen Psychiatry. 2007;64:1368-76.

72. Jablensky AV, Morgan V, Zubrick SR, Coger C, Yellachich LA. Pregnancy, delivery, and neonatal complications in a population cohort of women with schizophrenia and major affective disorders. Am J Psychiatry. 2005;162:79-91.

73. Gonzalez-Pinto A, Gutierrez M, Ezcurra J, Aizpuru F, Mosquera F, Lopez P, et al. Tobacco smoking and bipolar disorder. J Clin Psychiatry. 1998;59:225-8.

74. Morris CD, Giese AA, Turnbull JJ, Dickinson M, Johnson-Nagel N. Predictors of tobacco use among persons with mental illnesses in a statewide population. Psychiatr Serv. 2006;57:1035-8.

75. Weeke A, Vaeth M. Excess mortality of bipolar and unipolar manic-depressive patients. J Affect Disord. 1986;11:227-34.

76. Weeke A, Juel K, Vaeth M. Cardiovascular death and manicdepressive psychosis. J Affect Disord. 1987;13:287-92.

77. Saku M, Tokudome S, Ikeda M, Kono S, Makimoto K, Uchimura $\mathrm{H}$, et al. Mortality in psychiatric patients, with a specific focus on cancer mortality associated with schizophrenia. Int J Epidemiol. 1995;24:366-72.
78. Dutta R, Boydell J, Kennedy N, Van Os J, Fearon P, Murray RM. Suicide and other causes of mortality in bipolar disorder: a longitudinal study. Psychol Med. 2007;37:839-47.

79. Tsuang MT, Woolson RF, Fleming JA. Causes of death in schizophrenia and manic-depression. Br J Psychiatry. 1980;136:239-42.

80. Black DW, Winokur G, Nasrallah A. Mortality in patients with primary unipolar depression, secondary unipolar depression, and bipolar affective disorder: a comparison with general population mortality. Int J Psychiatry Med. 1987; 17:351-60.

81. Kallner G, Lindelius R, Petterson U, Stockman O, Tham A. Mortality in 497 patients with affective disorders attending a lithium clinic or after having left it Pharmacopsychiatry. 2000;33:813.

82. Høyer EH, Mortensen PB, Olesen AV. Mortality and causes of death in a total national sample of patients with affective disorders admitted for the first time between 1973 and 1993. Br J Psychiatry. 2000;176:76-82.

83. Evans NJ, Baldwin JA, Gath D. The incidence of cancer among in-patients with affective disorders. $\mathrm{Br} \mathrm{J}$ Psychiatry. 1974; 124:518-25. 